\title{
8.4 GHz VLBI observations of SN 2004et in NGC 6946
}

\author{
I. Martí-Vidal ${ }^{1}$, J. M. Marcaide ${ }^{1}$, A. Alberdi ${ }^{2}$, J. C. Guirado ${ }^{1}$, L. Lara ${ }^{2, \star}$, M. A. Pérez-Torres ${ }^{2}$, \\ E. $\operatorname{Ros}^{3}$, M. K. Argo ${ }^{4}$, R. J. Beswick ${ }^{4}$, T. W. B. Muxlow ${ }^{4}$, A. Pedlar ${ }^{4}$, I. I. Shapiro ${ }^{5}$, C. J. Stockdale ${ }^{6}$, \\ R. A. Sramek ${ }^{7}$, K. W. Weiler ${ }^{8}$, and J. Vinko ${ }^{9}$
}

1 Departament d'Astronomia i Astrofísica, Universitat de València, c/ Dr. Moliner 50, 46100 Burjassot, València, Spain e-mail: I.Marti-Vidal@uv.es

2 Instituto de Astrofísica de Andalucía, CSIC, c/ Camino bajo de Huétor 50, 18008 Granada, Spain

3 Max-Planck-Institut für Radioastronomie, Postfach 2024, 53010 Bonn, Germany

4 Jodrell Bank Observatory, Macclesfield, Cheshire SK11 9DL, UK

Harvard-Smithsonian Center for Astrophysics, 60 Garden St., MS 51, Cambridge, MA 02138, USA

6 Department of Physics, Marquette University, PO Box 1881, Milwaukee, WI 53201-1881, USA

National Radio Astronomy Observatory, PO Box O, Socorro, NM 87801, USA

8 Naval Research Laboratory, Code 7210, Washington, DC 20375-5320, USA

9 Department of Optics \& Quantum Electronics, University of Szeged, Szeged 6720, Hungary

Received 21 March 2007 / Accepted 22 May 2007

\section{ABSTRACT}

\begin{abstract}
We report on $8.4 \mathrm{GHz}$ very-long-baseline-interferometry (VLBI) observations of the type II-P supernova SN 2004et in the spiral galaxy NGC 6946, made on 20 February 2005 (151 days after explosion). The Very Large Array (VLA) flux density was $1.23 \pm$ $0.07 \mathrm{mJy}$, corresponding to an isotropic luminosity at $8.4 \mathrm{GHz}$ of $(4.45 \pm 0.3) \times 10^{25} \mathrm{erg} \mathrm{s}^{-1} \mathrm{~Hz}^{-1}$ and a brightness temperature of $(1.3 \pm 0.3) \times 10^{8} \mathrm{~K}$. We also provide an improved source position, accurate to about 0.5 mas in each coordinate. The VLBI image shows a clear asymmetry. From model fitting of the size of the radio emission, we estimate a minimum expansion velocity of $15700 \pm$ $2000 \mathrm{~km} \mathrm{~s}^{-1}$. This velocity is more than twice the expected mean expansion velocity estimated from a synchrotron self-absorbed emission model, thus suggesting that synchrotron self-absorption is not relevant for this supernova. With the benefit of an optical spectrum obtained 12 days after explosion, we favor an emission model which consists of two hot spots on an underlying expanding shell of width comparable to that of SN 1993J.
\end{abstract}

Key words. galaxies: individual: NGC 6946 - radio continuum: stars - stars: supernovae: individual: SN 2004et

\section{Introduction}

Supernova SN 2004et in galaxy NGC 6946 was discovered by Zwitter et al. (2004) on 27 September 2004. Yamaoka et al. (2004) reported data from the robotic Télescope à Action Rapide pour les Objects Transitoires (TAROT) telescope from which the supernova explosion date could be well constrained. Nothing brighter than magnitude $19.4 \pm 1.2$ was seen on September 22.017 and the supernova was detected at magnitude $15.17 \pm 0.16$ on September 22.983. Following Yamaoka et al., we assume 22.0 September 2004 as the explosion date.

NGC 6946 is a starburst spiral galaxy with a high supernova production rate. In fact, $\mathrm{SN} 2004 \mathrm{et}$ is the eighth supernova event registered in this galaxy since 1917 . The distance estimates to NGC 6946 range from 5.1 Mpc (de Vaucouleurs 1979) up to 10.1 Mpc (Sandage \& Tammann 1981). Here we adopt $5.5 \pm$ $0.1 \mathrm{Mpc}$ as the distance to NGC 6946 following Pierce (1994), who estimated it using the Tully-Fisher HI relation. Most of the distance values quoted in the literature vary between 5.2 and $5.5 \mathrm{Mpc}$. Sahu et al. (2006) derived a distance of 5.6 Mpc to NGC 6946 as an average of the literature values, including the result of their analysis for SN 2004et itself, which is in very good agreement with the adopted $5.5 \mathrm{Mpc}$ distance.

$\mathrm{Li}$ et al. (2005) found a progenitor candidate for this supernova by analyzing Canada-France-Hawaii Telescope (CFHT)

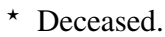

images of the field made before the supernova explosion. The progenitor seems to have been a yellow supergiant that may have previously experienced a red supergiant phase, but it could also have consisted of a binary system of red and blue supergiants. This latter case would imply a progenitor similar to that for SN 1993J (Van Dyk et al. 2002).

Spectroscopic observations of Zwitter et al. (2004) and Filippenko et al. (2004) suggest that SN 2004et is a type II supernova. Hence, according to the emission model of Chevalier (1982b), tested with other supernovae like SN 1993J (e.g., Marcaide et al. 1997), we expected that this supernova would emit detectable radio synchrotron radiation sometime after its optical detection. Indeed, the radio emission from SN 2004et was detected and monitored at $5 \mathrm{GHz}$ with the Multi-Element Radio Linked Interferometer Network (MERLIN) and the VLA arrays from October 2004 onwards (Argo et al. 2005; Kelley et al., in preparation). Monitoring was also carried out at $6.0 \mathrm{GHz}$ and $1.6 \mathrm{GHz}$ by these authors. Unfortunately, the monitoring observations by Argo et al. were unable to constrain the date of the $5 \mathrm{GHz}$ peak emission, because scheduling constraints meant that no observations could be made between 3-17 November 2004 (see Fig. 2 of Argo et al. 2005). From their observations, Argo et al. (2005) estimated the $5 \mathrm{GHz}$ peak flux density as $\sim 2.5 \mathrm{mJy}$ in early November 2004. Kelley et al. (in preparation), using observations of SN2004et at 6 different frequencies with the VLA, fitted the flux density evolution of the supernova to the 
"mini-shell" model of Chevalier (1982a,b) and obtained an estimate of the SN2004et peak flux density at $5 \mathrm{GHz}$ of $2.45 \pm 0.08 \mathrm{mJy}$ on day $66 \pm 6$ after explosion (assuming 22.0 September 2004 as the explosion date).

We arranged very-long-baseline-interferometry (VLBI) observations of SN 2004et in order to learn as much as possible about the radio emitting structure, the expansion scenario, and the emission mechanisms. In Sect. 2 we describe our observations; in Sect. 3 we present our VLBI image of SN 2004et and the results of fits of several emission models to the data; in Sect. 4 we discuss the magnetic field strength and the amount of energy involved in the radio emission, together with the expansion velocity of the emitting region and the importance of possible absorption mechanisms; in Sect. 5 we present our conclusions.

\section{Observations and data reduction}

\subsection{The VLBI data}

In our observations, made on 20 February 2005, the participating stations were the complete Very Long Baseline Array (VLBA) (10 identical antennae of $25 \mathrm{~m}$ diameter spread over the US from the Virgin Islands to Hawaii), the phased VLA (equivalent to $130 \mathrm{~m}$ diameter, New Mexico, USA), the Green Bank Telescope (100 m, West Virginia, USA), Goldstone (70 m, California, USA), Robledo (70 m, Madrid, Spain), Effelsberg (100 m, Bonn, Germany), and Medicina (32 m, Bologna, Italy).

The recording rate was $256 \mathrm{Mbps}$, with 2-bit sampling and single polarization mode, covering a total synthesized bandwidth of $64 \mathrm{MHz}$ (except at the phased VLA, where the effective bandwidth was $50 \mathrm{MHz}$ ). These data were correlated at the Array Operations Center of the National Radio Astronomy Observatory (NRAO) in Socorro (New Mexico, USA) with an averaging time of $2 \mathrm{~s}$.

The 12-h long, 8.4 GHz VLBI phase-reference observations were designed to maximize the probability for the detection of supernova SN 2004et. We used the source J2022+614 as the phase calibrator (a $3.0 \mathrm{Jy}$ quasar at an angular distance of $2.2^{\circ}$ from the supernova). We spent $25 \%$ of the observing time on the calibrator, with duty cycles of approximately 4 min. Every 22 min we also observed a secondary phase calibrator, the quasar J2035+582, with a 0.18 Jy total flux density and an angular separation of $1.76^{\circ}$ from the supernova.

The correlation coefficients were then read into the NRAO Astronomical Image Processing System (AIPS) for calibration purposes. A manual phase alignment between the 8 different IFs was obtained by fringe fitting a selected scan of the calibrator source $\mathbf{J} 2022+614$.

We then performed the amplitude calibration using gain curves for each antenna (information provided by NRAO and the European VLBI Network, EVN) and system temperatures measured by each station during the observations.

Such a scheme is appropriate for all stations except the phased VLA. In that case, the amplitude calibration requires previous knowledge of the flux densities of all sources. For consistency, we first mapped the calibrators using the VLBI data from all stations but the VLA. Then, we used the total fluxes obtained in the mapping of $\mathrm{J} 2022+614$ and $\mathrm{J} 2035+582$ to calibrate the phased VLA.

The J2022+614 data were then fringe-fitted in a standard manner and the resulting calibrated visibilities were exported to the Caltech program DIFMAP (Shepherd et al. 1995) for imaging purposes using standard phase (and later also amplitude)

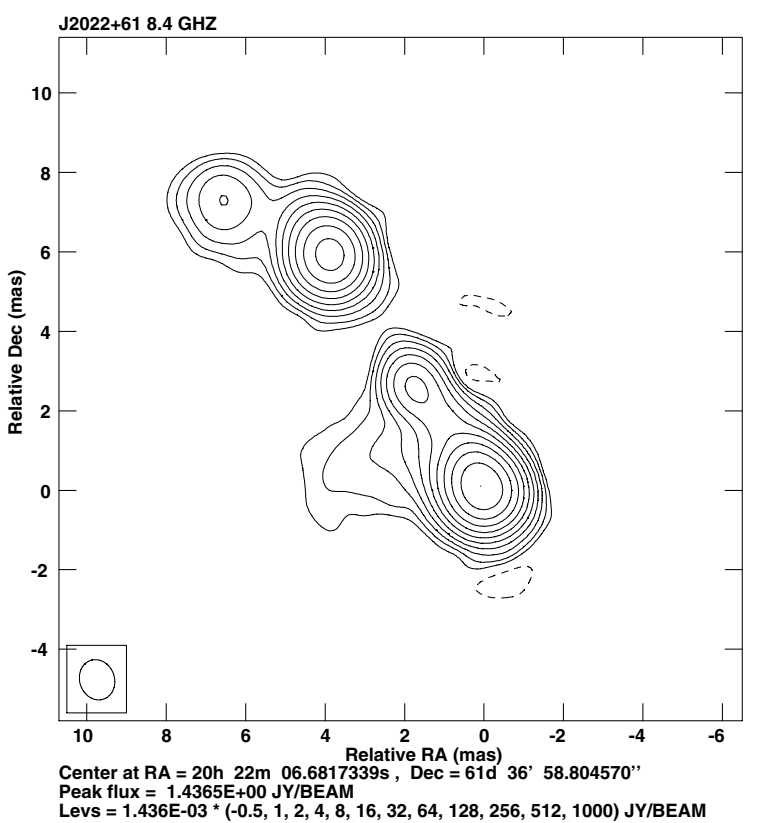

Fig. 1. Contour image of our phase calibrator source, J2022+614, at 8.4 GHz. The dynamic range (peak/rms) obtained for this image is 68 000:1. The FWHM of the CLEAN beam is shown at bottom left.

self-calibration techniques. The phase corrections derived from self-calibration differed from the corrections derived from fringe fitting by typically 5-10 degrees, and the amplitudes after selfcalibration differed from the system temperature calibrated amplitudes by typically $2-5 \%$. The resulting map of $\mathrm{J} 2022+614$ is shown in Fig. 1. Afterwards, the J2022+614 model obtained in DIFMAP was read back into AIPS for a second fringe-fitting in which we obtained residual phases, delays, and rate solutions nearly free of structure contributions.

These fringe-fitted solutions were then linearly interpolated to the times of the SN 2004et scans and were used for the calibration of the phases, delays and delay rates of the VLBI SN 2004et data. Finally, we exported the calibrated supernova data for hybrid mapping in DIFMAP.

\subsection{The VLA data}

The VLA provided two kinds of data: VLBI data from the phased array and individual antenna data. The correlation coefficients for all the pairs of antennas could thus be obtained and used for wide-field imaging around SN 2004et and for obtaining an accurate estimate of the total flux density of SN 2004et.

We also used J2022+614 as the flux calibrator for the VLA data. The radio image contours thus obtained are shown in Fig. 2 superimposed on an optical image. The map is affected by bandwidth smearing effects. The radio sources identified in Fig. 2 had previously been listed by Hyman et al. (2000). Since the correlation was centered on SN 2004et, its flux density was accurately determined as $1.23 \pm 0.07 \mathrm{mJy}$.

\section{VLBI image of SN 2004et}

In DIFMAP, we deleted the bad visibility amplitudes of SN 2004et (i.e., less than $0.1 \%$ of the data) and Fourier inverted the remaining data into the sky plane. We then applied the natural weighting scheme to the visibilities for sensitivity optimization and detected a nearly point emitter (showing a small 


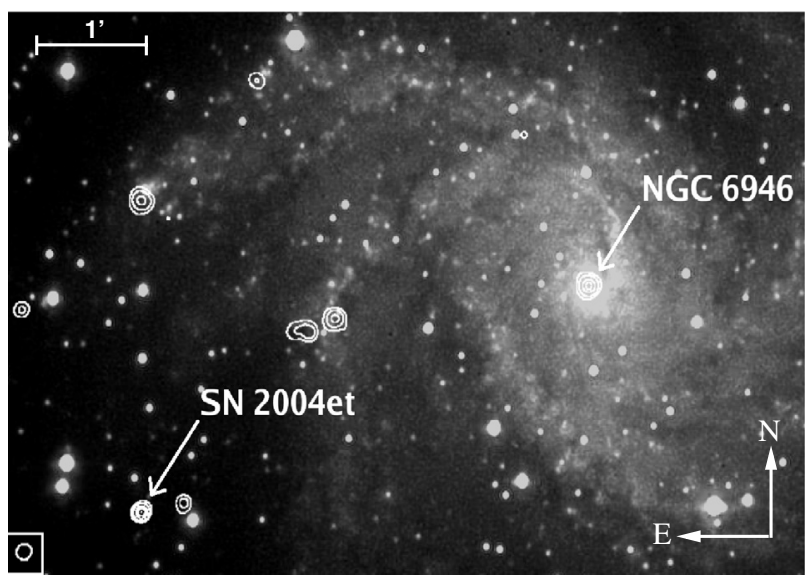

Fig. 2. Radio image contours from the VLA superimposed on an optical image of NGC 6946 (taken with the TROBAR telescope of the Observatori Astronòmic de la Universitat de València). The FWHM of the CLEAN beam is shown at bottom left. Contours are at 0.02, 0.08, $0.32,0.64$, and 1 times the peak flux density, which is $1.23 \mathrm{mJy} / \mathrm{Beam}$.

elongation in the South-East direction) whose peak was displaced 5.1 mas West and 14.7 mas South from the coordinates at which we had centered the correlation (we used the coordinates given by Beswick et al. 2004). The emitter is undoubtedly SN 2004et. Thus, a better position of the supernova, based on a phase-reference to $\mathrm{J} 2022+614$, a source of the International Celestial Reference Frame (ICRF), is: $\alpha=20^{\mathrm{h}} 35^{\mathrm{m}} 25.3596^{\mathrm{s}}$ and $\delta=+60^{\circ} 07^{\prime} 17.7203^{\prime \prime}$ (equinox J2000.0, $\pm 0.5 \mathrm{mas}^{1}$ in each coordinate).

Given the very low flux-density of SN 2004et, we did not apply any phase self-calibration to the data. We fitted several emission models to the real and imaginary parts of the visibilities using the DIFMAP task modelfit. The errors of the fitted parameters of the models correspond to the statistical uncertainties taken from the covariance matrices of the fits. A point source model fitted to the data has a total flux density of $0.63 \pm 0.03 \mathrm{mJy}$. After subtraction of this point source model from the data, there is still clear extra emission at the level of $0.24 \mathrm{mJy}_{\text {beam }}{ }^{-1}$, indicating structure in the supernova emission. A map of this extra emission is shown in Fig. 3.

This extra emission could be due to a bad antenna calibration, so we checked on this possibility by making a map of the source leaving out one antenna of the array at a time. We also imaged the first and second halves of these data separately to check the structure repeatability. All the resulting maps showed the extra emission when we subtracted a point source from the uv-data. Thus, we conclude that this emission belongs to SN 2004et and is not produced by imperfect calibration.

Using two point emitters to fit the data, we recovered a flux density of $(0.57 \pm 0.05) \mathrm{mJy}$ for the first component and $(0.26 \pm$ $0.05) \mathrm{mJy}$ for the second, located at an angular distance of $0.5 \pm$ 0.06 mas and at a position angle of $(136 \pm 4)^{\circ}$ with respect to the strongest component.

Given that the total recovered flux density in the uv data using CLEAN adds up to $0.87 \mathrm{mJy}$ (5\% higher than the flux density fitted), and the VLA flux density estimate is $1.23 \mathrm{mJy}$, there must be some missing flux density in our VLBI data. Most of this missing flux density could be due to a loss of coherence in the phase-referenced VLBI data compared to the VLA data.

1 This error corresponds to the beamwidth of the VLBI interferometer applying the natural weighting scheme to the SN 2004et visibilities.

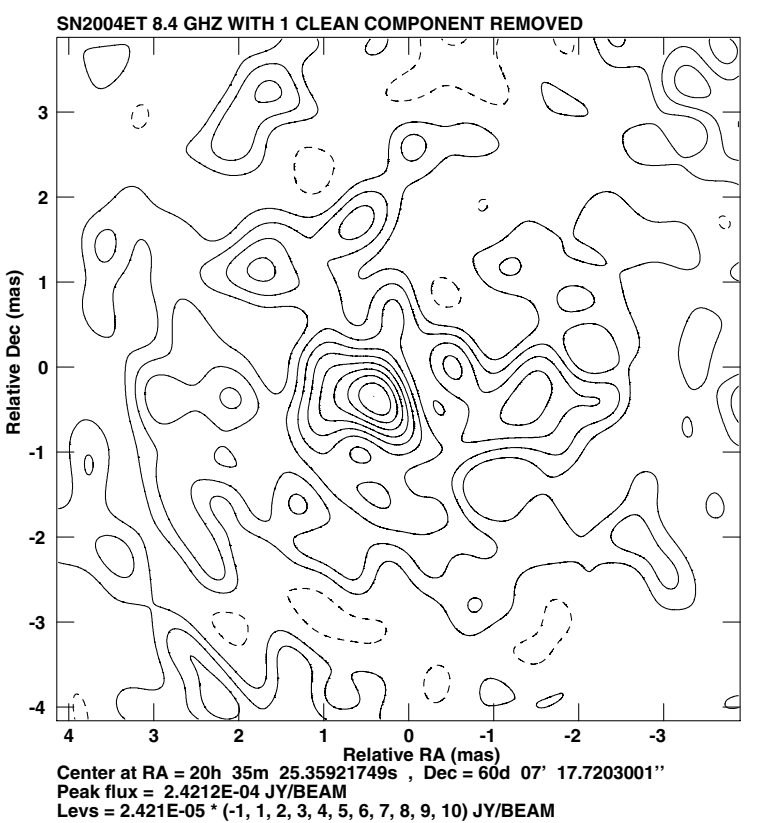

Fig. 3. Residuals after the subtraction of a single point source to SN 2004et (see text in Sect. 3).

Table 1. Results of different models fit to the SN 2004et data.

\begin{tabular}{cccc}
\hline \hline Model & $\begin{array}{c}\text { Diameter } \\
(\mathrm{mas})\end{array}$ & $\begin{array}{c}\text { Results } \\
\text { Total flux density } \\
(\mathrm{mJy})\end{array}$ & $\begin{array}{c}\text { rms of residuals } \\
(\mu \mathrm{Jy} / \mathrm{beam})\end{array}$ \\
\hline Uniform disk & $0.62 \pm 0.08$ & $0.81 \pm 0.05$ & 27 \\
Optically thin sphere & $0.8 \pm 0.1$ & $0.83 \pm 0.05$ & 27 \\
Optically thin shell $^{1}$ & $0.70 \pm 0.08$ & $0.82 \pm 0.05$ & 27 \\
Two point emitters $^{2}$ & $0.50 \pm 0.06$ & $0.83 \pm 0.07$ & 26 \\
\hline
\end{tabular}

1 The assumed shell width is $30 \%$ of the outer radius.

2 The diameter refers to the separation of the point emitters.

This part of the missing flux could be recovered using phase self-calibration, were the flux density of the source high enough. However, given that this is not the case, we decided to leave the phases unchanged and consider this lack of flux density as an instrumental limitation.

Disks or shell-like sources could also be used to fit the data. Indeed, we show the results of some of these fits in Table 1. We find that for the fit to two point emitters the resulting rms of the residuals is slightly (but not significantly) smaller than for any of the other fits, which are very similar. The similarity between the rms of the fits of all the different emission models indicates that the source is only barely resolved by the interferometer and it is not possible to distinguish between more detailed models.

We show the VLBI image of SN 2004et in Fig. 4. The peak brightness is $0.64 \mathrm{mJy} \mathrm{beam}^{-1}$ and the rms of the residuals $0.03 \mathrm{mJy}$ beam $^{-1}$.

\section{Discussion}

\subsection{Supernova energy and magnetic field}

The SN2004et flux density measured by the VLA on February 2005 using J2022+614 as the flux density calibrator is $1.23 \pm 0.07 \mathrm{mJy}$. For a distance to NGC 6946 of $5.5 \pm$ $0.1 \mathrm{Mpc}$, this flux density corresponds to an isotropic luminosity at $8.4 \mathrm{GHz}$ of $(4.5 \pm 0.3) \times 10^{25} \mathrm{erg} \mathrm{s}^{-1} \mathrm{~Hz}^{-1}$. The corresponding brightness temperature, if we use the size determined from 


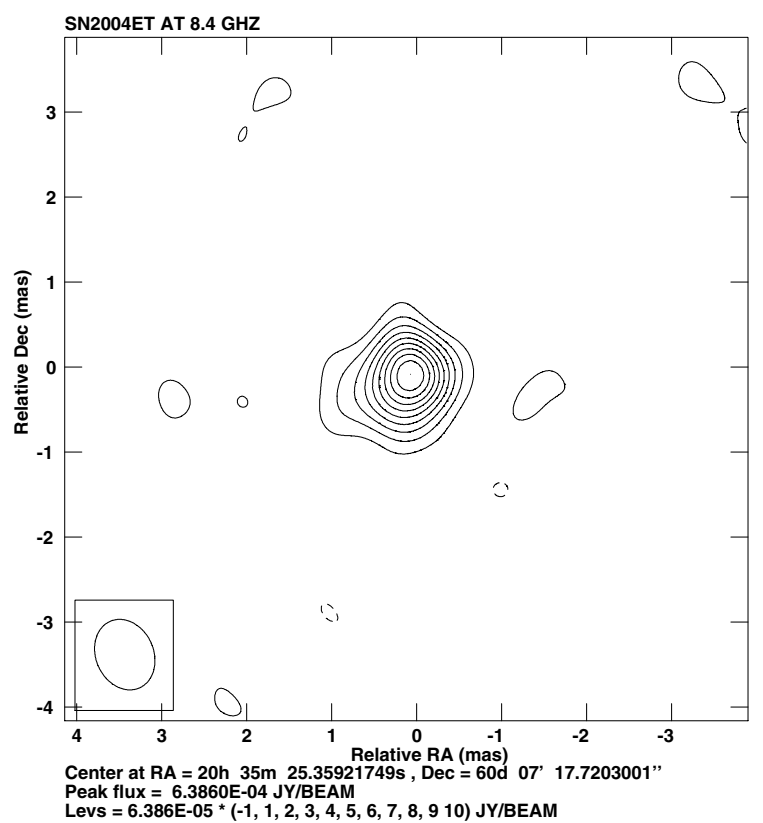

Fig. 4. Contour map of SN 2004et obtained from our VLBI data by model fitting two point sources (see text in Sect. 3). The lowest positive level shown is $10 \%$ of the peak, which is $0.64 \mathrm{mJy} / \mathrm{Beam}$. The FWHM of the CLEAN beam is shown at bottom left.

the fit to two point emitters, is $(1.3 \pm 0.3) \times 10^{8} \mathrm{~K}$, indicating a non-thermal synchrotron origin of the radio emission.

We did not obtain spectral information of the radio emission coming from SN2004et in our VLBI observations. However, Kelley et al. (in preparation) observed SN 2004et with the VLA at several frequencies (ranging from $1.4 \mathrm{GHz}$ to $43.3 \mathrm{GHz}$ ) during several hundred of days following the supernova explosion. These authors fit the evolution of the resulting radio light curves to a given parametric model, from which they estimate a spectral index, $\alpha=-0.87 \pm 0.04$, for the radio emission in the optically thin phase of the supernova evolution. Given that the supernova was in its optically thin phase during our VLBI observations, we used the spectral index estimated by Kelley et al. From the previously derived luminosity at $8.4 \mathrm{GHz}$, we estimate a total integrated radio luminosity of $(1.3 \pm 0.1) \times 10^{36} \mathrm{erg} \mathrm{s}^{-1}$ between 1.4 $\mathrm{GHz}$ and $43.3 \mathrm{GHz}$.

Using this result together with the SN 2004et size estimate from our VLBI observations, we can derive the minimum total energy in the radiating region of the supernova and the corresponding equipartition magnetic field. From Pacholczyk (1970) (see chapter 7 , formulae 14 and 15) we have:

$E_{\min }=c_{13}(1+k)^{4 / 7} \phi^{3 / 7} R^{9 / 7} L_{R}^{4 / 7}$

$B_{\text {eq }}=\left(4.5 c_{12}(1+k) / \phi\right)^{2 / 7} R^{-6 / 7} L_{R}^{2 / 7}$

where $c_{12}$ and $c_{13}$ are functions of the spectral index, $\alpha$, and the minimum and maximum frequencies considered in the spectrum integration, $\phi$ is the filling factor of the emitting region, $R$ is the source radius, $L_{R}$ is the integrated radio luminosity, and $k$ is the ratio between the heavy particle energy and the electron energy. The mechanism of generation is not well known, hence $k$ can vary from 1 to $m_{\mathrm{p}} / m_{\mathrm{e}} \approx 2 \times 10^{3}$. Thus, these formulae give us a range of possible values of the equipartition energy and magnetic field instead of an estimate. Also we do not know the value of the filling factor, $\phi$ (the fact that the emission may not be completely isotropic will also affect the estimate), but the effect of $\phi$ on the value of $E_{\min }$ and $B_{\text {eq }}$ goes aproximately as the square root and the cube root, respectively, as can be seen in Eqs. (1) and (2).

Assuming that the radio emitting region corresponds to a shell of width equal to $30 \%$ of the outer radius (e.g., Marcaide et al. 1995, 2007), we find that the filling factor, $\phi$, is 0.66 , and that the minimum total energy (particles plus fields) of the emitting region (with a radius of $0.70 \pm 0.08$ mas) ranges between $8.2 \times 10^{45} \mathrm{erg}$ and $4.22 \times 10^{47} \mathrm{erg}$. The upper part of the range still represents less than one part in a thousand of the supernova emission energy, $\sim 10^{51} \mathrm{erg}$.

The corresponding equipartition magnetic field ranges between $37 \mathrm{mG}$ and $260 \mathrm{mG}$. This range is similar to those obtained for other type II supernovae, like SN 1986J (Pérez-Torres et al. 2002), SN 1993J (e.g., Pérez-Torres et al. 2001) or, more recently, SN 2001gd (Pérez-Torres et al. 2005). Values in this range are very high compared to the typical (about $1 \mathrm{mG}$ ) magnetic fields in the circumstellar medium of a massive star. Given that the hydrodynamic compression from the supernova shock only increases the circumstellar magnetic field by a factor of about 4 (e.g., Dyson \& Williams 1980), other field amplification mechanisms, i.e. turbulent amplification possibly due to Rayleigh-Taylor instabilities (Chevalier 1982b), must be taking place in the emitting region of SN 2004et.

\subsection{Supernova structure}

Our results show that the emission structure of supernova SN 2004et is not spherically symmetric. The residuals of the fit of a point emitter to the VLBI data, shown in Fig. 3, indicate that the emitting structure has an angular asymmetry.

The structure of the ejecta of type II-P supernovae are supposed to be very spherical, given that their extended hydrogen envelope should "spherize" the possible asymmetries in the shock (e.g., Khokhlov et al. 2001). Thus, the asymmetry found in SN 2004et is likely to come from the structure of the circumstellar material.

Assuming a circumstellar origin for the asymmetry of the SN 2004et emission, we may interpret such asymmetry in two ways:

Model 1. A shell with two hot spots, one on each "side" of the emission of the shell. These hot spots could be due to anisotropies of the circumstellar density distribution (probably due to asymmetries in the progenitor pre-supernova wind) and/or anisotropies of the magnetic field. This kind of anisotropic shell has been found in the case of SN 1986J (Bartel et al. 1991; Pérez-Torres et al. 2002; Bietenholz et al. 2004). The fact that we recovered a much lower flux density with VLBI than with the VLA is consistent with this model. The two hot spots of the radio structure would emit the major part of the total flux density of the source, and the emission from the shell, being faint, would be buried in the thermal noise of the map. A fluxdensity distribution with a peak surface brightness just under $0.1 \mathrm{mJy} \mathrm{beam}^{-1}$ (peak flux density of the noise; see the spread contours of Fig. 3) distributed over an area of 2 VLBI beams (roughly, the area of the radio structure), would account for a flux density of $\sim 0.2 \mathrm{mJy}$, that is, half of the difference between the flux density recovered by the VLA and VLBI.

Model 2. An expanding shell plus a protrusion. This protrusion could be produced by an asymmetry in the circumstellar medium distribution, like an axi-symmetric region with a lower circumstellar density. Such asymmetry could result in a faster expansion of the portion of the forward shock located in that region. According to hydrodynamical simulations 
(Blondin et al. 1996), the length of the protrusion could be more than twice the radius of the main shell, but the time required for the formation of such a structure in the forward shock would be of the order of several years, much larger than the age of SN 2004et at the time of our observations.

Given the youth of the supernova (151 days at the epoch of our observations), the size of the protrusion should be much smaller than the mean size of the shell. This argument does not favour Model 2 as the origin of the observed structure.

\subsection{Expansion velocity}

The different models shown in Table 1 yield different estimates of the size of SN 2004et. We do not know which one of these models (or another) best represents the true emitting structure of SN 2004et. Thus, we will estimate the minimum expansion velocity compatible with our data using the smallest of our size estimates. This separation (see Table 1) is $0.50 \pm 0.06$ mas and corresponds to the model of two compact emitters.

Assuming a distance of $5.5 \pm 0.1 \mathrm{Mpc}$ for NGC 6946 and 22.0 September 2004 as the explosion date of the supernova, we find that the radial distance between the point components translates into a mean separation speed of $31400 \pm 4000 \mathrm{~km} \mathrm{~s}^{-1}$ between them. Assuming also that the two compact emitters come from two diametrically opposed hot spots on an expanding shell, the center of the explosion would fall half way between them and the expansion velocity of the shell would be $15700 \pm$ $2000 \mathrm{~km} \mathrm{~s}^{-1}$. This velocity is high compared with the theoretical predictions of a synchrotron self-absorbed model, as will be shown below.

If we use the size estimates of the other models in Table 1, the derived expansion velocities are higher than $15700 \mathrm{~km} \mathrm{~s}^{-1}$. Fitting the data to a disk with uniform emission (unrealistic, because the circumstellar region is not optically thick at this epoch), yields an expansion speed of the ejecta of $19500 \pm$ $2500 \mathrm{~km} \mathrm{~s}^{-1}$; fitting to an optically thin shell-like model yields a speed $22000 \pm 2500 \mathrm{~km} \mathrm{~s}^{-1}$, and fitting to an optically thin filled sphere results in a speed of $25200 \pm 3000 \mathrm{~km} \mathrm{~s}^{-1}$.

An early optical spectrum of SN 2004et is shown in Fig. 5 and shows characteristic P Cygni profiles of an expanding envelope. This spectrum was taken on 3 October 2004 (7 days after discovery and 12 days after explosion) at the David Dunlap Observatory in Canada with the Cassegrain spectrograph on the 74 inch $(188 \mathrm{~cm})$ telescope as part of observations that will be fully described by Vinko et al. (in preparation). From the position of the blue absorption minimum of the $\mathrm{H} \alpha$ line, an expansion velocity of the line of $11900 \pm 600 \mathrm{~km} \mathrm{~s}^{-1}$ is estimated, once the redshift of the host galaxy is corrected. This velocity represents the velocity of the $\mathrm{H} \alpha$ "photosphere", i.e. where the optical depth becomes $\tau \sim 1$. Because at early phases the ejecta are almost fully ionized, this layer should be close to the top of the ejecta. Thus, the velocity from the $\mathrm{H} \alpha$ line should also be close to (although somewhat lower than) the expansion velocity of the ejecta.

Sahu et al. (2006) have reported expansion speeds from optical observations starting on day 30 after explosion (25 days after discovery). They identify a low velocity component in $\mathrm{H} \alpha$, which is the same as that shown in Fig. 5 but for later days and hence at lower velocities. They also identify a high velocity component for days 30 through 90 . Such high velocity feature is expected to arise from the interaction between the CSM and the SN shock wave (Chugai et al. 2007). It corresponds to a feature placed on the absorption side of the P Cygni profile of the lower velocity $\mathrm{H} \alpha$, both in their spectra and in the spectrum shown in

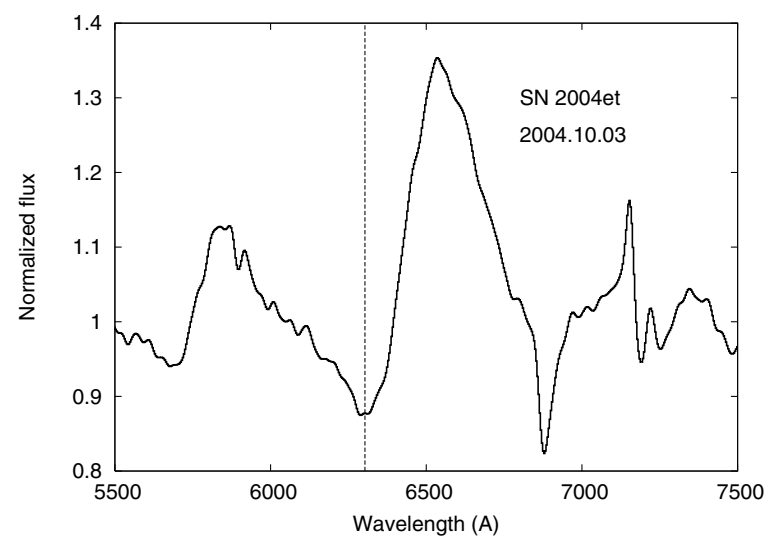

Fig. 5. Red part of an optical spectrum of SN 2004et obtained at the David Dunlap Observatory (see Vinko et al., in preparation). The spectrum is normalized to the continuum. The vertical dashed line marks the position of the blue absorption minimum of $\mathrm{H} \alpha$, corresponding to the velocity of the $\mathrm{H} \alpha$ "photosphere" (see text).

Fig. 5. However, this component appears very weak and almost imperceptible in Fig. 5. We are not convinced that it provides a good estimate of the early expansion speed and we will not use hereafter. Also, Chugai et al. (2007) did not find convincing evidence for the high-velocity $\mathrm{H} \alpha$ feature in the middle and late plateau phases, when such features should be more pronounced.

According to the model of Chevalier (1982b), the optical emission from young supernovae originates in the shocked ejecta region, located behind the shocked circumstellar region. This implies that the maximum velocities inferred from the optical lines should always be smaller than the velocities inferred from the VLBI observations of the radio emitting structure. The latter radio emission would originate in the shocked circumstellar region. In fact, according to the self-similar expansion model of Chevalier (1982a,b), the ratio between the maximum velocity inferred from the optical spectral lines in the very early days and the expansion velocity of the radio shell should be similar to the ratio between the inner and the outer radii of the shell-like radio emitting structure. Marcaide et al. (2007) find a ratio of $0.69 \pm$ 0.02 between the inner and the outer radii of the shell-like structure of supernova SN 1993J.

For SN 2004et we find that the ratio between the maximum velocity inferred from the optical spectral lines in the very early days and the expansion velocity of the radio shell is about 0.76 for the model yielding the smallest expansion speeds from the radio data. Thus, if the components of the two-point model are hot spots on the outer part of an underlying shell (which we do not detect, as explained above, due to its faintness) the width of such a shell would be comparable to that of SN 1993J. In arriving at this conclusion, we have not considered the deceleration of the supernova ejecta between the epoch of the optical observations and the epoch of the VLBI observations. This neglect implies that the optical line velocity at our VLBI epoch could be lower than $11900 \mathrm{~km} \mathrm{~s}^{-1}$. Thus, also taking into account that $15700 \mathrm{~km} \mathrm{~s}^{-1}$ is the smallest expansion speed estimated from these radio data, we conclude that the real ratio between the inner and the outer radii of the supernova radio shell could be somewhat smaller than 0.76 .

For cases of supernovae where synchrotron self-absorption (SSA) dominates over the circumstellar free-free absorption, and where there is equipartition of the energy between relativistic particles and magnetic fields, Chevalier (1998) found a relationship between the observing frequency, the size of the emitting 
region, the peak intensity of the supernova light curve, the supernova age at the peak, and the spectral index of the energy distribution of the relativistic electrons. For such a case, one can estimate the expansion velocity of a supernova based on measurements of the peak luminosity at a given frequency, the supernova age at the peak, and the synchrotron spectral index, as follows:

$$
\begin{aligned}
V_{\mathrm{SSA}}\left(\mathrm{km} \mathrm{s}^{-1}\right)= & 5.3786 \times 10^{6}(\beta \phi)^{-0.0541}\left(\frac{F_{\mathrm{p}}}{1 \mathrm{Jy}}\right)^{0.4729} \\
& \times\left(\frac{D}{1 \mathrm{Mpc}}\right)^{0.9458}\left(\frac{v}{1.0 \mathrm{GHz}}\right)^{-1}\left(\frac{t_{\mathrm{p}}}{1 \text { day }}\right)^{-1}
\end{aligned}
$$

where $\beta$ is the ratio of the relativistic particle energy density to the magnetic field energy density (since we assume energy equipartition, $\beta=\frac{4}{3(1+k)}$, Pacholczyk 1970, chapter 7), $\phi$ is the filling factor of the emitting region for a sphere (we assume a shell of width equal to $30 \%$ of the outer radius, which yields $\phi=0.66), F_{\mathrm{p}}$ is the flux density at the peak, $D$ is the distance, $v$ is the observing frequency, and $t_{\mathrm{p}}$ is the age at the peak. We use the synchrotron spectral index found by Kelley et al. (in preparation) to set the index of the electron energy distribution to $\gamma=2.74$ (the energy distribution of the relativistic electrons is assumed to be of the form $N \propto E^{-\gamma}$ and $\gamma=1-2 \alpha$ ).

When we use Eq. (3) and assume for $F_{\mathrm{p}}$ the $5 \mathrm{GHz}$ peak flux density published by Argo et al. (2005) (i.e., $F_{\mathrm{p}}=2.5 \mathrm{mJy}$ at $t_{\mathrm{p}}=$ 42 days), we estimate a SSA expansion velocity of the radio shell of SN 2004et of $9700 \pm 1800 \mathrm{~km} \mathrm{~s}^{-1}$. This is much lower than the estimate for each of the models in Table 1. If instead we use the $5 \mathrm{GHz}$ peak flux density fitted by Kelley et al. (in preparation) at day $66 \pm 6$ after explosion, which appears more accurate given the extended time coverage of their observations near the emission peak at $5 \mathrm{GHz}$, the SSA expansion velocity of the radio shell is only $6100 \pm 1100 \mathrm{~km} \mathrm{~s}^{-1}$. This result is less than half of any of the estimates based on our VLBI data.

As noted by Chevalier (1998), if the free-free absorption from the circumstellar medium is comparable to, or even higher than, the synchrotron self-absorption, the peak of emission will be defined by the free-free absorption evolution. This will produce a negative bias in the estimate of the ejecta expansion velocity, as we seem to find for SN 2004et. Comparison of VLBI results and radio light curves for other supernovae also gives important negative biases (see Chevalier 1998), but at present there is an insufficient number of cases (in fact, only SN 1979C, SN 1986J, and SN 1993J) to reach firm conclusions about the typical values of these biases. For supernovae SN 1986J and $\mathrm{SN} 1979 \mathrm{C}$, the bias ratios $V_{\mathrm{VLBI}} / V_{\mathrm{SSA}}(\sim 2.56$ and $\sim 2.60$, respectively) are similar to that for SN 2004et (if we use the minimum size compatible with our data), but for SN 1993J this bias ratio is smaller $(\sim 1.5)$.

\subsection{Comparison with other supernovae in NGC 6946}

From those eight supernovae known to have exploded in NGC 6946, only four have been detected as radio supernovae: SN 1968D, SN 1980K, SN 2002hh, and SN2004 et. For two (SN 1980K and SN 20004et), it has been possible to fit the light curves (Weiler et al. 1992; Kelley et al., in preparation) in terms of the "mini-shell" model (Chevalier 1982a,b).

There are a number of similarities between SN 1980K and SN 2004et. The flux-density power-law time decay parameter takes the values -0.62 and -0.74 , respectively, and the powerlaw parameter of the evolution of the external opacity with time takes the values -2.46 and -3.07 , respectively. But there are also significant differences between these supernovae: the spectral index is -0.52 for SN 1980K, while for SN 2004et it is -0.87 ; the time after explosion needed to reach the peak spectral luminosity was $134 \pm 2$ days for SN $1980 \mathrm{~K}$ and is only $66 \pm 6$ days for SN 2004et. For the case of SN 1980K (Weiler et al. 1992), internal and clumpy opacity contributions are not required to obtain a good fit, and the light curves are well characterized by an optically thin synchrotron radio emission absorbed by an uniform external medium ${ }^{2}$. However, for SN 2004et (see Kelley et al., in preparation), the absorption produced by a uniform external medium cannot fit the data, but a good fit is obtained for absorption by a clumpy or filamentary external medium. The presence of this clumpy external medium could explain the asymmetry in the radio structure that we have found in our VLBI image. On the one hand, an expansion into a clumpy medium would yield an irregular interaction between the ejecta and the circumstellar medium and, thus, the evolution of the expanding material would be asymmetric. On this account, Model 2 would appear supported. On the other hand, the synchrotron emission, being proportional to the relativistic electron density in the circumstellar region, would be larger in the clumps. Thus, these clumps in the CSM would give rise to several hot spots in the expanding radio shell and would appear to give support to Model 1. The asymmetry found in our VLBI image is, then, consistent with the expansion of the supernova ejecta into a clumpy medium.

\section{Conclusions}

We report on $8.4 \mathrm{GHz}$ VLBI observations of supernova SN 2004et in NGC 6946. The observations, made 151 days after explosion, are the first VLBI observations of a type II-P supernova, and successfully detect the radio emission and yield an image of the source. This image shows evidence of structure.

This structure has a geometry that could be interpreted either as emission coming from a shell with two hot spots (Model 1) or emission coming from a region expanding anisotropically and developing a protrusion (Model 2). However, such a protrusion would be too large for so young a supernova, to be compatible with the hydrodynamic simulations of Blondin et al. (1996).

The asymmetry found in our VLBI image can be explained if the supernova expands in the clumpy circumstellar medium suggested by the fits to the radio light curves of this supernova (Kelley et al., in preparation).

The minimum expansion speed that we obtain is $15700 \pm$ $2000 \mathrm{~km} \mathrm{~s}^{-1}$ for a two point emitter model. Using other models yields larger expansion speeds. Maximum velocity estimates obtained from optical spectral line observations give lower values than the velocities inferred from our VLBI data for all cases. Such differences between maximum optical and radio expansion speeds can be understood in the framework of the self-similar expansion model of Chevalier (1982a,b). In particular, the ratio of the maximum early $\mathrm{H} \alpha$ line velocity (corresponding in the Chevalier model to the maximum velocity of the ejecta) to the expansion velocity estimated from VLBI for a two point model is $\sim 0.76$, similar to that for SN 1993J. This ratio suggests that Model 1 is a plausible model.

In any case, the expansion speed derived from our VLBI data is more than twice larger than the estimate obtained using the synchrotron self-absorbed model proposed by Chevalier (1998). This discrepancy indicates that the synchrotron self-absorption

\footnotetext{
2 For SN 1968D, it has not been possible to fit the light curves due to the late detection (at an age of $\sim 26$ years), but the measured flux densities are consistent with the extrapolated radio behaviour of SN 1980K.
} 
must be negligible (i.e., small or nonexistent) compared to the circumstellar free-free absorption in SN 2004et at our observing epoch.

The flux density obtained from our VLA data $(1.23 \mathrm{mJy})$ and our minimum VLBI size estimate (diameter of 0.5 mas) translate into a brightness temperature of $(1.3 \pm 0.3) \times 10^{8} \mathrm{~K}$, indicating a synchrotron origin for the radiation. Using the spectral index $\alpha=-0.87 \pm 0.04$, determined by Kelley et al. (in preparation) with data between 1.4 and $43.3 \mathrm{GHz}$, we find that the amount of energy injected into the emitting region is in the range $(8-420) \times$ $10^{45} \mathrm{erg}$, a small fraction (lower than 0.001 ) of the typical emission energies in supernova explosions. The equipartition magnetic field falls within (37-260) $\mathrm{mG}$, indicating the existence of highly efficient field amplification due perhaps to turbulent instabilities in the emitting region.

Further VLBI observations of this supernova are not possible given its low flux density, now well below the VLBI sensitivity limit at the possible data rates at present. VLBI observations of similar, type II-P, supernovae will be essential for the understanding of the details of the radio emission of this kind of explosion.

Acknowledgements. This work has been partially funded by Grants AYA200422045-E, AYA2005-08561-C03, and AYA2006-14986-C02 of the Spanish DGICYT. The National Radio Astronomy Observatory is a facility of the National Science Foundation operated under cooperative agreement by Associated Universities, Inc. The European VLBI Network is a joint facility of European, Chinese, South African and other radio astronomy institutes funded by their national research councils. K.W.W. thanks the Office of Naval Research for the 6.1 funding supporting this research. J. V. received support from Hungarian OTKA Grants No. TS 049872 and T042509. We thank the Observatori Astronòmic of the UV for obtaining the optical image of NGC 6946. We thank the anonymous referee for his/her useful comments.

\section{References}

Argo, M. K., Beswick, R. J., Muxlow, T. W. B., et al. 2005, MmSAI, 76, 565 Bartel, N., Rupen, M. P., Shapiro, I. I., et al. 1991, Nature, 350, 212 Beswick, R. J., Muxlow, T. W. B., Argo, M. K., et al. 2004, IAUC, 8435, 3 Bietenholz, M. F., Bartel, N., \& Rupen, M. 2004, Science, 304, 1947 Blondin, J. M., Lundqvist, P., \& Chevalier, R. A. 1996, ApJ, 472, 257 Chevalier, R. A. 1982a, ApJ, 258, 790

Chevalier, R. A. 1982b, ApJ, 259, 302

Chevalier, R. A. 1998, ApJ, 499, 810

Chugai, N. N., Chevalier, R. A., \& Utrobin, V. P. 2007, [arXiv:astro-ph/0703468]

Dyson, J. E., \& Williams, D. A. 1980, Physics of the Interstellar Medium (New York: Wiley)

Filippenko, A. U., Foley, R. J., Treu, T., et al. 2004, IAUC, 8414

Hyman, S. D., Lacey, C. K., Weiler, K. W., et al. 2000, AJ, 119, 1711

Khokhlov, A., Höflich, P., \& Wang, L. 2001, in 20th Texas symposium on relativistic astrophysics (American Institute of Physics), 586

Li, W., Van Dyk, S. D., Filippenko, A. V., et al. 2005, PASP, 117, 121

Marcaide, J. M., Alberdi, A., Ros, E., et al. 1995, Nature, 373, 44

Marcaide, J. M., Alberdi, A., Ros, E., et al. 1997, ApJ, L486, 31

Marcaide, J. M., et al. 2007, in preparation

Pacholczyk, A. G. 1970, Radio Astrophysics (San Francisco: Freeman)

Pérez-Torres, M. A., Alberdi, A., \& Marcaide, J. M. 2001, A\&A, 374, 997

Pérez-Torres, M. A., Alberdi, A., \& Marcaide, J. M. 2002, MNRAS, 335, 23

Pérez-Torres, M. A., Alberdi, A., Marcaide, J. M., et al. 2005, MNRAS, 360, 1055

Pierce, M. J. 1994, ApJ, 430, 53

Sahu, D. K., Anupama, G. C., Srividya, S., et al. 2006, MNRAS, 372, 1315

Sandage, A., \& Tammann, G. A. 1981, A Revised Shapley-Ames Catalog of Bright Galaxies (Carnegie Inst. Washington Publ.), 635

Shepherd, M. C., Pearson, T. J., \& Taylor, G. B. 1995, BAAS, 26, 987

Van Dyk, S. D., Garnavich, P. M., Filippenko, A. V., et al. 2002, PASP, 114, 1322 de Vaucouleurs, G. 1979, ApJ, 227, 729

Weiler, K. W., Van Dyk, S. D., Panagia, N., et al. 1992, ApJ, 398, 248

Yamaoka, H., Itagaki, K., Klotz, A., et al. 2004, IAUC, 8413, 2

Zwitter, T., Munari, U., \& Moretti, S. 2004, IAUC, 8413, 1 